\title{
DETERMINING THE CONTACT AREA IN ELASTIC-INDENTATION PROBLEMS
}

\section{J. R. BARBER Department of Mechanical Engineering, University of Newcastle upon Tyne}

A general relationship is established, determining the contact area in the indentation of an elastic half-space.

Contact pressure is shown to increase monotonically with load throughout the contact area.

\section{INTRODUCTION}

It is well known that, with a suitable choice of auxiliary solution, the reciprocal theorem can be used to yield simple relations between load and displacement for elastic bodies in situations for which a full solution would be difficult or impossible. (See, for example, (1)*) In a recent paper Shield (2) shows how this technique can be applied to problems of indentation of the elastic semi-infinite solid by a rigid punch. The load-displacement relation can be written as an integral over the punch profile provided that we know (1) the extent of the contact area which is established and (2) the stress distribution which would be developed under a flat rigid punch covering this same area and impressed a unit distance into the elastic solid.

If the punch has a continuous profile, it is not generally possible to determine the extent of the contact area from purely geometrical considerations, though it may be possible to determine its shape from considerations of symmetry as with the axi-symmetric punch. For this latter case, Sneddon (3) and Shield (2) have derived expressions which enable the radius of the contact area to be found from considerations of continuity of stress at its boundary.

In this paper a more general theorem is proved which determines the contact area for any punch, provided the shape of the contact area can be specified in terms of a limited number of parameters. As an illustration, it is shown how expressions can be obtained for the semi-axes of the elliptical contact area established in the Hertzian contact problem without the making of any assumption as to the distribution of stress over this area.

\section{PRELIMINARY CONSIDERATIONS}

We suppose the punch to be smooth, in which case there will be no tangential stress on the surface plane $(z=0)$, and we can represent the stresses and displacements in the solid $(z>0)$ in terms of a single harmonic function $\phi(x, y, z)$ such that at the surface (4)

$$
\left.\begin{array}{l}
u_{z}=-\frac{(1-\nu)}{G} \partial \phi / \partial z \\
\sigma_{z z}=-\partial^{2} \phi / \partial z^{2}
\end{array}\right\}
$$

The function $\partial \phi / \partial z$ is thus mathematically analogous with the electrostatic potential due to a distribution of charge

$$
q=\sigma_{z z} / 2 \pi
$$

The MS. of this paper was received at the Institution of Mechanica Engineers on 11th March 1974 and accepted for publication on 19th July 1974. 33

*References are given in the Appendix. per unit area on the plane $z=0$ in an otherwise empty space (5).

We shall require the following theorem of potential theory which is conveniently stated in the terminology of electrostatics (6):

In the field due to a self-equilibrating system of charges, the maximum potential must occur in a region of positive charge whilst the minimum potential must occur in a region of negative charge.

Since all the charge occurs on the surface plane, it is clear from the above analogy that the maximum and minimum normal surface displacements $\left(u_{z}\right)$ must occur in regions where $\sigma_{z z}$ is respectively negative and positive, provided that the integral of $\sigma_{z z}$ over the surface plane is zero. This condition can always be satisfied by placing a force at infinity to keep the solid in equilibrium. However, if this is done, either the maximum or the minimum value of $u_{z}$ (but not both) may occur at infinity according to the direction of the net force on the solid in the finite domain

\section{THE CONTACT PROBLEM}

We now suppose that a rigid punch of arbitrary shape is pressed into the solid to a prescribed depth, establishing a contact area $A_{0}$. The boundary conditions on the surface ( $z$ $=0)$ are

$$
\left.\begin{array}{ll}
u_{z}=f(x, y) & \text { on } A_{0} \\
\sigma_{z z}=0 & \text { outside } A_{0}
\end{array}\right)
$$

where $f$ is a prescribed function.

The extent of the contact area is determined from the conditions

$$
\left.\begin{array}{lr}
\boldsymbol{o}_{z z} \leqslant 0 & \text { on } A_{0} \\
u_{z}>f(x, y) & \text { outside } A_{0}
\end{array}\right\}
$$

Now let $A$ represent any area (not necessarily simply connected) on the surface of the solid and let $P(A)$ be defined by

$$
P(A)=-\iint_{A} \sigma_{z z}(A) \mathrm{d} A
$$

where $\sigma_{z z}(A)$ is determined from the boundary conditions (3) with $A$ written in place of $A_{0}$. We shall prove the following theorem.

The maximum value of $P(A)$ occurs when $A \equiv A_{0}$; i.e. when conditions (4) are satisfied.

Proof. Consider the effect of increasing $A$ by a small element $\delta A$. 
If $P(A)$ is thereby increased the corresponding differential contact-stress distribution amounts to a net compressive force and hence, by the theorem of the previous section, the maximum differential displacement $\delta u_{z}$ must occur somewhere in $A$ or $\delta A$. However, $\delta u_{z}$ is zero throughout $A$, since $f(x, y)$ is uniquely prescribed. The maximum value of $\delta u_{z}$ cannot be zero since, ex hypothesi, the differential stress system represents a net force in the $z$ direction; hence the maximum must occur in $\delta A$ and the contact stress there must be compressive.

We know that the final value of $u_{z}$ in $\delta A$ is $f(x, y)$. The effect of the differential contact-stress distribution has thus been shown to be an increase of $u_{z}$ at $\delta A$ to the value $f(x$, $y)$ and it follows that $\delta A$ is an area for which initially

$$
u_{z}<f(x, y)
$$

Next, suppose there is some other area $\delta A^{\prime}$ within $A$, the release of which involves an increase in $P(A)$. Again the differential contact-stress distribution causes zero displacement throughout that part of $A$ which remains in contact $\left(A-\delta A^{\prime}\right)$, whilst the maximum $\delta u_{z}$ occurs in $\delta A^{\prime}$. It follows that the differential stress in $\delta A^{\prime}$ must be compressive and, since this leads to a final condition of zero contact stress on $\delta A^{\prime}$, this area must originally have been one on which

$$
o_{z z}>0
$$

Hence, an increase in $P(A)$ can only be achieved by altering $A$ if there are some areas outside $A$ satisfying condition (6) or inside $A$ satisfying condition (7). It follows that the maximum value of $P(A)$ occurs when $A \equiv A_{0}$; i.e. when conditions (4) are satisfied.

This proof does not depend upon the profile of the punch being continuous, the contact stress being bounded at the edge of the contact area or the contact area being connected.

\section{THE EFFECT OF PENETRATION DEPTH}

Theorem. The contact area $A_{0}$ and the contact pressure at any point within it increase monotonically with load.

Proof: Suppose a given contact area $A_{0}$ is established by a load $P . P$ is now increased by $\delta P$, with a resultant increase in contact area $\delta A_{0}$, whilst any region within $A_{0}$ tending to leave contact is restrained by tensile stress.

As in section 3 , we can prove that the maximum differential displacement $\delta u_{z}$ must occur in the new contact area $\left(A_{0}+\delta A_{0}\right)$ and from geometrical considerations it must occur in $A_{0}$ (over which $\delta u_{z}$ is constant) since there is initially clearance between the punch and the surface in $\delta A_{0}$.

Now if the differential stress were tensile at any point on $A_{0}$, it would follow from equation (1) that in such a region

$$
-\frac{\partial^{2} \phi}{\partial z^{2}}>0
$$

In other words $(-\partial \phi / \partial z)$ would increase with $z$ as we moved into the solid from an admittedly maximum value, which is self-contradictory. Hence there can be no tensile differential contact stress at any point on $A_{0}$.

It follows that there is no point within $A_{0}$ at which the contact pressure falls as a consequence of increasing $P$ and, as a special case, there is no region at which contact is broken (by the contact stress falling to zero).

\section{EXAMPLE}

We can apply the theorem of section 3 to the determination of the semi-axes of the elliptical contact area in the Hertzian contact problem. Using Shield's method to find $P(A)$ we require as auxiliary solution the stress distribution necessary to produce a unit displacement over the ellipse $A$ bounded by

$$
\frac{x^{2}}{a^{2}}+\frac{y^{2}}{b^{2}}-1=0 . . \quad . \quad . \quad . \quad . \quad . \quad . \quad . \quad .
$$

This is derived by Green and Sneddon (7) and is

$$
\sigma_{z z}=-\frac{G\left(1-x^{2} / a^{2}-y^{2} / b^{2}\right)^{-1 / 2}}{(1-\nu) b \mathrm{~K}\left(\sqrt{1-b^{2} / a^{2}}\right)}
$$

where

$$
K(k)=\int_{0}^{\pi / 2} \frac{d x}{\sqrt{1-k^{2} \operatorname{Sin}^{2} x}}
$$

If the punch profile is defined by

$$
f(x, y)=d-\alpha x^{2}-\beta y^{2}
$$

where $d$ is the depth of penetration, we can apply the reciprocal theorem to equations (10) and (12) to obtain

$$
\begin{aligned}
& P(A)=-\iint_{A}\left(\sigma_{z z} \times 1\right) \mathrm{d} A \\
& =-\iint_{A}-\frac{G\left(1-x^{2} / a^{2}-y^{2} / b^{2}\right)^{-1 / 2} \times\left(d-\alpha x^{2}-\beta y^{2}\right)}{(1-\nu) b \mathrm{~K}\left(\sqrt{\left.\left(1-b^{2} / a^{2}\right)\right)}\right.} \mathrm{d} A \\
& =\frac{2 \pi G a}{(1-\nu) \mathrm{K}\left(\sqrt{\left.1-b^{2} / a^{2}\right)}\right.}\left\{d-8 / 3\left(\alpha a^{2}+\beta b^{2}\right)\right\}
\end{aligned}
$$

The semi-axes $a, b$ can now be obtained from the two equations

$$
\frac{\partial P(A)}{\partial a}=0 ; \quad \frac{\partial P(A)}{\partial b}=0
$$

after which $p$ can be found by substituting back into equation (13).

This method makes no assumption about the distribution of contact pressure other than that the contact area is an ellipse. If $\alpha=\beta$, the conditions degenerate into that given by Shield (2) for the axi-symmetric punch.

\section{DISCUSSION}

For the classical contact problems of elasticity, the theorem proved in section 3 furnishes a criterion for determining the extent of the contact area which is an alternative to and equipollent with the more usual specification of continuity of contact pressure at the edge of the area. However, the latter method is subject to two limitations

(1) The profile of the punch must be continuous in the first derivative at all points which might form part of the boundary of the contact region, since otherwise the 
contact pressure may be legitimately discontinuous at the boundary. A simple example of this type is indentation by a truncated conical punch.

(2) The contact area must be connected. If this condition is not satisfied, it will be possible to find invalid solutions which nonetheless satisfy the condition of continuity at the boundary of the contact area. Thus, if at some stage in the application of the load a second discrete contact area is established, the continuity condition is insufficient to distinguish between the true solution and the solution in which the increment in load is entirely carried by a further enlargement of the original contact area.

The method described in this paper is not subject to either of these limitations and is to that extent more general in its analytic application.

Neither method is capable of determining directly the exact boundary of the contact region for an arbitrary contact problem, since both methods require that this boundary should be already specified in terms of a limited number of parameters, e.g., the semi-axes $a, b$ in the example of section 5 .

However, a wide range of punch profiles might be expected to give an approximately elliptical contact area, the dimensions and orientation of which could be estimated by use of the theorem of section 3. This use of the theorem is analogous to the familiar application of energy methods in other problems of elasticity, i.e. an approximate mode of deformation is defined in terms of a limited number of parameters, best values of which are then determined from energy considerations.
Finally, a numerical approach to the general contact problem based on the theorem could be developed by dividing the possible contact area into a limited number of discrete elements. The function $P(A)$, equation (5), could be determined for any prescribed subset of these elements by solving a set of simultaneous equations. The determination of the true elastic contact area is thus transformed to an optimization problem for this function.

By contrast, the argument from continuity of contact pressure does not lend itself to approximate or numerical applications.

\section{APPENDIX \\ REFERENCES}

(1) TIMOSHENKO, S. P. and GOODIER, J. N. Theory of elas. ticity 3rd edition $1970 \$ 97$ (McGraw-Hill Book Co. Inc., New York and London).

(2) SHIELD, R. T. 'Load-displacement relations for elastic bodies', Z. Agnew. Math. Phys. 1967 18, 682-93.

(3) SNEDDON, I. N. 'The relation between load and penetration in the axi-symmetric Boussinesq problem for a punch of arbitrary profile', Int. J. Engng Sci, $19653,47-57$.

(4) GREEN, A. E. and ZERNA, W. Theoretical elasticity 1954 $171-2$ (Clarendon Press, Oxford).

(5) GREEN, A. E. 'On Bousinesq's problem and penny-shaped cracks', Proc. Cambridge Phil. Soc. 1949 45, 251-7.

(6) MAXWELL, J. C. Electricity and magnetism 3rd edition 1892 $\S 112$ (Clarendon Press, Oxford).

(7) GREEN, A. E. and SNEDDON, 1. N. 'The distribution of stress in the neighbourhood of a flat elliptical crack in an elastic solid', Proc. Cambridge Phil. Soc. 1950 46, 159 64. 